\title{
Two short proofs concerning tree-decompositions
}

\author{
Patrick Bellenbaum \& Reinhard Diestel
}

\begin{abstract}
We give short proofs of the following two results: of Thomas's theorem that every finite graph has a linked tree-decomposition of width no greater than its tree-width, and of the 'tree-width duality theorem' of Seymour and Thomas that the tree-width of a finite graph is exactly one less than the largest order of its brambles.
\end{abstract}

\section{Introduction}

The main purpose of this note is to give a short proof of Thomas's theorem that every finite graph has a linked tree-decomposition of width no greater than its tree-width [9]. This is a useful tool in the theory of tree-decompositions; for example, it is a key lemma in Robertson \& Seymour's proof of the fact that every set of graphs of bounded tree-width is well-quasi-ordered [7]. This latter result is the starting point for the proof of their graph minor theorem, see [2]. It is also the first step in the now available short proof of the 'general Kuratowski theorem' that embeddability in any fixed surface is characterized by finitely many forbidden minors (combine it with [3] and either [5] or [10]), a main corollary of the graph minor theorem.

Another (more constructive) short proof of Thomas's theorem has been given in [1]. An analogous result for branch-width was obtained by Geelen, Gerards \& Whittle [4], also with a short and simple proof. The 'branch-width' of a graph is a parameter closely related to tree-width but not 1-1 translatable, so the result proved in [4] does not imply Thomas's theorem as reproved in this paper. But [4] does give a complete short proof (including the WQO part) of the above-mentioned result from [7], where exact bounds for Thomas's theorem are not required.

Our proof of Thomas's theorem differs from the original in that we use a simpler induction parameter. This simplifies the induction step: we have less to verify, and the presentation becomes considerably less technical. The tree-decomposition we use, however, is the same as in [9]. Essentially, it is constructed recursively from tree-decompositions of two subgraphs by adding their intersection as a new part to both decompositions, to serve as the common interface required for their amalgamation. Whether or not this can be done without increasing the width is a key issue in the study of tree-decompositions more generally. Thomas's sufficient condition under which this technique can be applied is a contribution of independent use and interest, and so we have extracted it into a separate lemma (Lemma 2).

This lemma (with some inessential additional details) has been used again before, in Seymour \& Thomas's proof of what Reed [6] has called their treewidth duality theorem [8]. A streamlined proof of this result has already ap- 
peared in [2], with the lemma incorporated. Since we need the lemma by itself in our different context here, we take the opportunity to include the short derivation of the duality theorem from it as well (Theorem 5).

All the graphs we consider are finite. Unless otherwise specified our terminology follows [2], and we assume familiarity with the basic theory of treedecompositions as covered there. In particular, we shall freely use the following separation lemma:

Lemma 1. Let $\left(V_{t}\right)_{t \in T}$ be a tree-decomposition of a graph $G$, let $t_{1} t_{2}$ be an edge of $T$, and let $T_{1}$ and $T_{2}$ be the components of $T-t_{1} t_{2}$. Then $V_{t_{1}} \cap V_{t_{2}}$ separates $\bigcup_{t \in T_{1}} V_{t}$ from $\bigcup_{t \in T_{2}} V_{t}$ in $G$.

\section{Amalgamating tree-decompositions}

Let $G$ be a graph and $X \subseteq V(G)$ a separating set of vertices. A basic technique in the study of tree-decompositions is to try to amalgamate given tree-decompositions of the subgraphs $H=G[C \cup X]$, where $C$ ranges over the components of $G-X$, into an overall tree-decomposition of $G$. This is straightforward if $X$ is contained in a part in each of those decompositions. If $X$ is not contained in a part of the given decomposition of $H$, we can alter that decomposition and force $X$ to become included in a part; to maintain the decomposition axiom (T3), however, this will typically involve the inclusion of the vertices from $X$ in some of the other parts as well, which can increase the width of that decomposition.

The lemma we prove in this section offers a sufficient condition for when $X$ can be incorporated into a part of a given decomposition of $H$ without increasing its width. The decomposition of $H$ will be given as induced by another tree-decomposition $\mathcal{D}$ of all of $G$, and the sufficient condition will involve the position of $X$ within $\mathcal{D}$.

Slightly more generally let $C$ be a union of components of $G-X$ and $H:=G[C \cup X]$. Let $\mathcal{D}=\left(V_{t}\right)_{t \in T}$ be a tree-decomposition of $G$, and $s \in T$. For each $x \in X$ pick a 'home' node $t_{x} \in T$ with $x \in V_{t_{x}}$, and for all $t \in T$ put

$$
W_{t}:=\left(V_{t} \cap V(H)\right) \cup\left\{x \in X \mid t \in t_{x} T s\right\} .
$$

Clearly $X \subseteq W_{s}$, and it is easy to check that $\mathcal{D}_{s}(H):=\left(W_{t}\right)_{t \in T}$ is a treedecomposition of $H$. (In fact, it is the tree-decomposition obtained from the decomposition which $\mathcal{D}$ induces on $H$ by forcing $X \subseteq W_{s}$ and repairing (T3) with the minimum addition of vertices to existing parts.)

Lemma 2. If $G-C$ contains a set $\left\{P_{x} \mid x \in X\right\}$ of disjoint $X-V_{s}$ paths with $x \in P_{x}$ for all $x$, then $\left|W_{t}\right| \leqslant\left|V_{t}\right|$ for all $t \in T$.

Proof. For each $x \in X$ with $x \in W_{t} \backslash V_{t}$ we have $t \in t_{x} T s$, so $V_{t}$ separates $V_{t_{x}}$ from $V_{s}$ by Lemma 1 and hence contains some other vertex of $P_{x}$. That vertex does not lie in $W_{t}$, because $W_{t} \backslash X \subseteq V(C)$ while $P_{x} \subseteq G-C$. 


\section{Linked and lean tree-decompositions}

A tree-decomposition $\mathcal{D}=\left(V_{t}\right)_{t \in T}$ is called linked if, in addition to the usual axioms (T1)-(T3), it satisfies the following:

(T4) Given any $k \in \mathbb{N}$ and $t_{1}, t_{2} \in T$, either $G$ contains $k$ disjoint $V_{t_{1}}-V_{t_{2}}$ paths or there exists a $t \in t_{1} T t_{2}$ such that $\left|V_{t}\right|<k$.

Let us call the decomposition $\mathcal{D}$ lean if, in addition to (T1)-(T3), it satisfies

(T4') Given $t_{1}, t_{2} \in T$ and vertex sets $Z_{1} \subseteq V_{t_{1}}$ and $Z_{2} \subseteq V_{t_{2}}$ such that $\left|Z_{1}\right|=\left|Z_{2}\right|=$ : $k$, either $G$ contains $k$ disjoint $Z_{1}-Z_{2}$ paths or there exists an edge $t t^{\prime} \in t_{1} T t_{2}$ with $V_{t} \cap V_{t^{\prime}}<k$.

It is easy to make a lean tree-decomposition linked without increasing its width: just subdivide every edge $t t^{\prime} \in T$ by a new node $s$ and add $V_{s}:=V_{t} \cap V_{t^{\prime}}$ as a new part. The greater freedom in choosing the sets $Z_{1}$ and $Z_{2}$ in $\left(\mathrm{T} 4^{\prime}\right)$, however, makes (T4') considerably stronger than (T4), especially when $t_{1}=t_{2}$. (For example, the trivial decomposition into one part is always linked, but not lean unless $G$ is complete.) More examples illustrating the difference between linked and lean decompositions, including a justification of term 'lean' based on this difference, can be found in [1].

Theorem 3. Every graph $G$ has a lean tree-decomposition of width $\operatorname{tw}(G)$, the tree-width of $G$.

Proof. Let $n:=|G|$. Let the fatness of a tree-decomposition of $G$ be the $n$-tuple $\left(a_{0}, \ldots, a_{n-1}\right)$, where $a_{h}$ denotes the number of parts that have exactly $n-h$ vertices. Let $\mathcal{D}=\left(V_{t}\right)_{t \in T}$ be a tree-decomposition of lexicographically minimal fatness. Clearly $\mathcal{D}$ has width $\operatorname{tw}(G)$; we shall prove that $\mathcal{D}$ is lean.

Suppose not. Then there exists a quadruple $\left(t_{1}, t_{2}, Z_{1}, Z_{2}\right)$ as in $\left(\mathrm{T} 4^{\prime}\right)$ that violates $\left(\mathrm{T} 4^{\prime}\right)$; we choose one for which $t_{1}$ and $t_{2}$ have minimum distance in $T$. Among all the $Z_{1}-Z_{2}$ separators of minimum order in $G$ let $X$ be one that lies 'closest to $t_{1} T t_{2}$ ' in the sense that $\sum_{x \in X} d_{x}$ is minimum, where

$$
d_{x}:=\min \left\{d_{T}\left(t, t_{x}\right) \mid t \in t_{1} T t_{2} \text { and } x \in V_{t_{x}}\right\} .
$$

Let $C_{1}$ denote the union of those components of $G-X$ that meet $Z_{1}$, let $C_{2}$ be the union of all the other components of $G-X$, and put $H_{i}:=G\left[C_{i} \cup X\right]$ for $i=1,2$. By Menger's theorem, there exists a set $\left\{P_{x} \mid x \in X\right\}$ of disjoint $Z_{1}-Z_{2}$ paths in $G$ such that $x \in P_{x}$ for all $x$. Each $P_{x}$ is the union of two paths $P_{x}^{i} \subseteq G-C_{i}$ meeting exactly in $x$; the paths $P_{x}^{i}$ will be used below to apply Lemma 2.

For every $x \in X$ choose $t_{x}$ at minimum distance from $t_{1} T t_{2}$ in $T$ so that $x \in V_{t_{x}}$. Let $T^{1}, T^{2}$ be disjoint copies of $T$. For $i=1,2$ and $t \in T$ let $t^{i}$ denote the copy of $t$ in $T^{i}$, and put $T^{\prime}:=T^{1} \cup T^{2}+t_{2}^{1} t_{1}^{2}$. Let $\mathcal{D}_{t_{3-i}}\left(H_{i}\right)=:\left(W_{t}^{i}\right)_{t \in T}$ be the tree-decomposition of $H_{i}$ obtained from $\mathcal{D}$ for $s:=t_{3-i}$ as before Lemma 2 . 
Rewriting $T$ as $T^{i}$ and $W_{t}^{i}$ as $W_{t^{i}}$ we may combine $\mathcal{D}_{t_{2}}\left(H_{1}\right)$ and $\mathcal{D}_{t_{1}}\left(H_{2}\right)$ to a tree-decomposition $\mathcal{D}^{\prime}=\left(W_{t}\right)_{t \in T^{\prime}}$ of $G$. (Note that $\mathcal{D}^{\prime}$ satisfies (T3), because $\left.V\left(H_{1} \cap H_{2}\right)=X \subseteq W_{t_{1}^{2}} \cap W_{t_{2}^{1}}.\right)$

To complete the proof we show that $\mathcal{D}^{\prime}$ has smaller fatness than $\mathcal{D}$. To do so, we prove that

and

$$
(\forall t \in T)(\forall i=1,2)\left(\left|W_{t}^{i}\right|=\left|V_{t}\right| \Rightarrow W_{t}^{3-i} \subseteq X\right)
$$

$$
\left(\exists t \in t_{1} T t_{2}\right)\left(\left|W_{t}^{1}\right|,\left|W_{t}^{2}\right|<\left|V_{t}\right|\right) .
$$

To see why this suffices, note that $\left|W_{t}^{i}\right| \leqslant\left|V_{t}\right|$ by Lemma 2 , and recall that since $\mathcal{D}$ violates $\left(\mathrm{T} 4^{\prime}\right)$ we have $|X|<k \leqslant\left|V_{t}\right|$ for all $t \in t_{1} T t_{2}$. Thus (1) implies that for every $h>|X|$ the number of parts of order $h$ is no greater in $\mathcal{D}^{\prime}$ than it was in $\mathcal{D}$, while (2) implies that for some such $h$ this number has gone down.

For the proof of (1) let $t \in T$ be given, assume for notational simplicity that $i=1$, and suppose that $\left|W_{t}^{1}\right|=\left|V_{t}\right|$; we show that $W_{t}^{2} \subseteq X$. If not then $V_{t}$ meets $C_{2}$, and for every vertex of $V_{t}$ in $C_{2}$ some $x \in X$ was included in $W_{t}^{1}$ when it was formed from $V_{t}$. Let $Y$ be the set of all those $x$ :

and put

$$
Y:=W_{t}^{1} \backslash V_{t}=\left\{x \in X \mid t \in t_{x} T t_{2}\right\} \backslash V_{t},
$$

$$
X^{\prime}:=(X \backslash Y) \cup\left(V_{t} \cap C_{2}\right)
$$

(Fig. 1). As indicated above, $\left|W_{t}^{1}\right|=\left|V_{t}\right|$ implies that $|Y|=\left|V_{t} \cap V\left(C_{2}\right)\right|$, so $\left|X^{\prime}\right|=|X|<k$. Our aim is to show that $X^{\prime}$ should have been chosen instead of $X$ for our minimal counterexample.

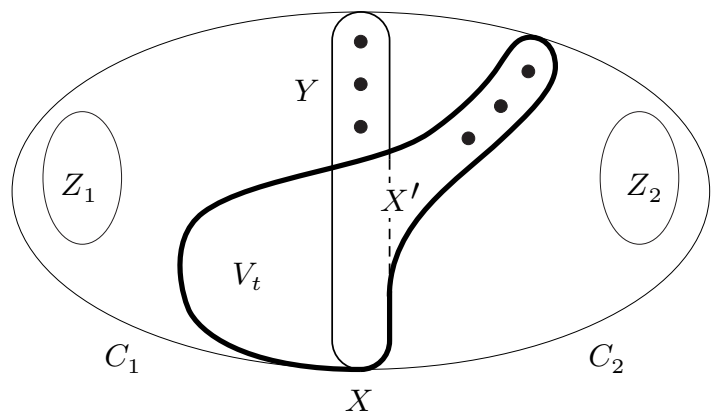

Figure 1. Obtain $X^{\prime}$ from $X$ by replacing the vertices of $Y$ with vertices from $V_{t} \cap V(C)$.

To this end, let us show that $X^{\prime}$ separates both $Z_{1}$ and $V_{t}$ from $Z_{2}$ in $G$. Any path $P$ in $G-X^{\prime}$ from either $Z_{1}$ or $V_{t}$ to $Z_{2}$ has a last vertex $y$ in $Y$. Then $\stackrel{\circ}{y} \subseteq C_{2}$, and $y P$ contains a $V_{t_{y}}-V_{t_{2}}$ path. Since $t \in t_{y} T t_{2}$ by $(*), y P$ meets $V_{t}$ (Lemma 1), and as $y \notin V_{t}$ it must do so in $C_{2}$, ie. in $X^{\prime}$. 
If $t \in \stackrel{\circ}{t}_{1} T t_{2}$ then the above implies that for any $k$-set $Z \subseteq V_{t}$ the quadruple $\left(t, t_{2}, Z, Z_{2}\right)$ violates $\left(\mathrm{T} 4^{\prime}\right)$, which contradicts the choice of $t_{1}$ and $t_{2}$. So $t \notin \grave{t}_{1} T t_{2}$. We complete the proof of (1) by showing that $d_{x^{\prime}}<d_{y}$ for all $x^{\prime} \in X^{\prime} \backslash X$ and all $y \in Y\left(=X \backslash X^{\prime}\right)$; then $X^{\prime}$ should have been chosen instead of $X$ for our counterexample $\left(t_{1}, t_{2}, Z_{1}, Z_{2}\right)$.

Let $x^{\prime}$ and $y$ be given. By $(*) t$ separates $t_{y}$ from $t_{2}$ in $T$, and hence as $t \notin \stackrel{\circ}{1}_{1} T t_{2}$ from the whole path $t_{1} T t_{2}$. Writing $d$ for the distance of $t$ from $t_{1} T t_{2}$ in $T$, we thus obtain $d_{x^{\prime}} \leqslant d<d_{y}$ by $x^{\prime} \in V_{t}$ and the definition of $t_{y}$.

For the proof of (2) it suffices by (1) to find $t \in t_{1} T t_{2}$ such that $V_{t}$ crosses $X$, ie. meets both $C_{1}$ and $C_{2}$. Suppose there is no such $t$. Since $V_{t_{1}}$ meets $C_{1}$ and $V_{t_{2}}$ meets $C_{2}$, this means that $t_{1} T t_{2}$ has an edge $t t^{\prime}$ such that $V_{t} \subseteq H_{1}$ and $V_{t^{\prime}} \subseteq H_{2}$. But then $V_{t} \cap V_{t^{\prime}} \subseteq X$ and $t t^{\prime}$ satisfies (T4'), a contradiction.

\section{The tree-width duality theorem}

Let $G$ be a graph. Two subsets of $V(G)$ are said to touch if they have a vertex in common or $G$ contains an edge between them. Following [6], we call a set $\mathcal{B}$ of mutually touching connected vertex sets a bramble. A subset of $V(G)$ is said to cover $\mathcal{B}$ if it meets every element of $\mathcal{B}$. The least number of vertices covering a bramble is its order. A typical example of an order $n$ bramble is the set of crosses in the $n \times n$ grid; see [2].

Lemma 4. Any set of vertices separating two covers of a bramble also covers that bramble.

Proof. Since each set in the bramble is connected and meets both of the covers, it also meets any set separating these covers.

Theorem 5. Let $k \geqslant 0$ be an integer. A graph has tree-width $\geqslant k$ if and only if it contains a bramble of order $>k$.

Proof. For the backward implication, let $\mathcal{B}$ be any bramble in a graph $G$. We show that every tree-decomposition $\left(V_{t}\right)_{t \in T}$ of $G$ has a part that covers $\mathcal{B}$. For every edge $e \in T$, at least one of the two components $T^{\prime}$ of $T-e$ is such that $\bigcup_{t \in T^{\prime}} V_{t}$ covers $\mathcal{B}$, because the sets in $\mathcal{B}$ are connected and touch (cf. Lemma 1); we then orient the edge $e$ towards $T^{\prime}$. Having oriented every edge of $T$ we let $s$ be the last vertex of a maximal directed path in $T$ and note that $V_{s}$ covers $\mathcal{B}$.

To prove the forward direction, we now assume that $G$ contains no bramble of order $>k$. We show that for every bramble $\mathcal{B}$ in $G$ there is a $\mathcal{B}$-admissible tree-decomposition of $G$, one in which any part of order $>k$ fails to cover $\mathcal{B}$. For $\mathcal{B}=\emptyset$ this implies that $\operatorname{tw}(G)<k$, because every set covers the empty bramble.

Let $\mathcal{B}$ be given, and assume inductively that for every bramble $\mathcal{B}^{\prime}$ containing more sets than $\mathcal{B}$ there is a $\mathcal{B}^{\prime}$-admissible tree-decomposition of $G$. (The 
induction starts, since no bramble in $G$ has more than $2^{|G|}$ sets.) Let $X \subseteq V(G)$ be a cover of $\mathcal{B}$ with as few vertices as possible; then $\ell:=|X| \leqslant k$ is the order of $\mathcal{B}$. Our aim is to show the following:

For every component $C$ of $G-X$ there exists a $\mathcal{B}$-admissible tree-decomposition of $G[C \cup X]$ with $X$ as a part.

Then these tree-decompositions can be combined to a $\mathcal{B}$-admissible tree-decomposition of $G$ by identifying their nodes corresponding to $X$. (If $X=V(G)$, then the tree-decomposition with $X$ as its only part is $\mathcal{B}$-admissible.)

So let $C$ be a fixed component of $G-X$, write $H:=G[C \cup X]$, and put $\mathcal{B}^{\prime}:=\mathcal{B} \cup\{C\}$. If $\mathcal{B}^{\prime}$ is not a bramble then $C$ fails to touch some element of $\mathcal{B}$, and hence $Y:=V(C) \cup N(C)$ does not cover $\mathcal{B}$. Then the tree-decomposition of $H$ consisting of the two parts $X$ and $Y$ satisfies $(*)$.

So we may assume that $\mathcal{B}^{\prime}$ is a bramble. Since $X$ covers $\mathcal{B}$, we have $C \notin \mathcal{B}$ and hence $\left|\mathcal{B}^{\prime}\right|>|\mathcal{B}|$. Our induction hypothesis therefore ensures that $G$ has a $\mathcal{B}^{\prime}$-admissible tree-decomposition $\mathcal{D}=\left(V_{t}\right)_{t \in T}$. If this decomposition is also $\mathcal{B}$-admissible, there is nothing more to show. If not, then one of its parts of order $>k, V_{s}$ say, covers $\mathcal{B}$. Since no set of fewer than $\ell$ vertices covers $\mathcal{B}$, Lemma 4 implies with Menger's theorem that $V_{s}$ and $X$ are linked in $G$ by $\ell$ disjoint paths $P_{x}$ with $x \in P_{x}$ for all $x \in X$. As $V_{s}$ fails to cover $\mathcal{B}^{\prime}$ and hence lies in $G-C$, so do these paths.

For each $x \in X$ pick a 'home' node $t_{x} \in T$ with $x \in V_{t_{x}}$, and consider $\mathcal{D}_{s}(H)=\left(W_{t}\right)_{t \in T}$ as in Lemma 2. Then $W_{s}=X$. To complete the proof of $(*)$, we show that $\mathcal{D}_{s}(H)$ is $\mathcal{B}$-admissible. Consider any $W_{t}$ of order $>k$. Then $W_{t}$ meets $C$, because $W_{t} \subseteq V(H)$ and $|X| \leqslant k$. Since $\mathcal{D}$ is $\mathcal{B}^{\prime}$-admissible and $\left|V_{t}\right| \geqslant\left|W_{t}\right|>k$ by Lemma 2 , we know that $V_{t}$ fails to meet some $B \in \mathcal{B}$; let us show that $W_{t}$ does not meet this $B$ either. If it does, it must do so in some $x \in X$ with $x \in W_{t} \backslash V_{t}$. Then $B$ is a connected set meeting both $V_{s}$ and $V_{t_{x}}$ but not $V_{t}$, contradicting $t \in s T t_{x}$ by Lemma 1 .

Theorem 5 can be restated in terms of the bramble number of a graph, the largest order of any bramble in it. It then says that the tree-width of a graph is exactly one less than its bramble number. 


\section{References}

[1] P. Bellenbaum, Schlanke Baumzerlegungen von Graphen, Diplomarbeit Universität Hamburg 2000.

[2] R. Diestel, Graph theory, 2nd edition, Springer-Verlag 2000 and http://www.math. uni-hamburg. de/home/diestel/books/graph.theory/download.html

[3] R. Diestel, K.Yu. Gorbunov, T.R. Jensen \& C. Thomassen, Highly connected sets and the excluded grid theorem, J. Combin. Theory B 75 (1999), 61-73. (Also included in [2] with improved exposition.)

[4] J.F. Geelen, A.M.H. Gerards \& G. Whittle, Branch width and well-quasiordering in matroids and graphs, J. Combin. Theory B 84 (2002), 270-290.

[5] J.F. Geelen, B. Richter, \& G. Salazar, Embedding grids in surfaces, preprint 2000.

[6] B.A. Reed, Tree-width and tangles: a new connectivity measure and some applications, in (R.A. Bailey, ed) Surveys in Combinatorics 1997, Cambridge University Press 1997, 87-162.

[7] N. Robertson \& P.D. Seymour, Graph minors IV; tree-width and wellquasi-ordering, J. Combin. Theory B 48 (1990), 227-254.

[8] P.D. Seymour \& R. Thomas, Graph searching and a min-max theorem for tree-width, J. Combin. Theory B 58 (1993), 22-33.

[9] R. Thomas, A Menger-like property of tree-width; the finite case, J. Combin. Theory B 48 (1990), 67-76.

[10] C. Thomassen, A simpler proof of the excluded minor theorem for higher surfaces, J. Combin. Theory B 70 (1997), 306-311. 